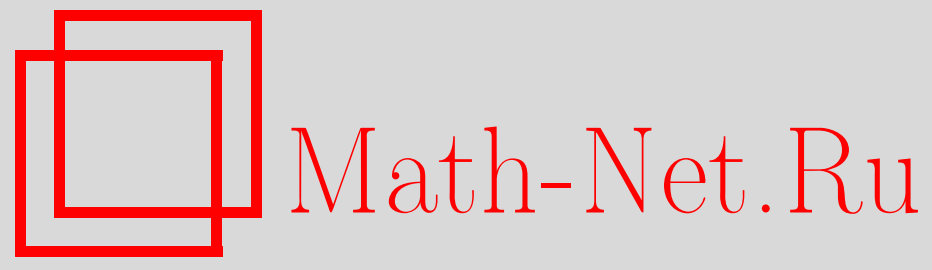

Л. В. Степанова, Е. М. Адылина, Асимптотические методы нелинейной механики разрушения: результаты, современное состояние и перспективы, Вестн. Сам. гос. техн. ун-та. Сер. Физ.-мат. науки, 2013, выпуск 2(), 156-168

DOI: https://doi.org/10.14498/vsgtu1156

Использование Общероссийского математического портала Math-Net.Ru подразумевает, что вы прочитали и согласны с пользовательским соглашением

http: //www . mathnet.ru/rus/agreement

Параметры загрузки:

IP : 54.92 .164 .108

26 апреля 2023 г., 18:21:28

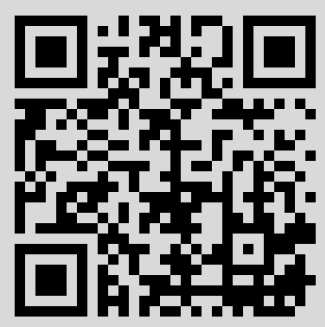


Вестн. Сам. гос. техн. ун-та. Сер. Физ.-мат. науки. 2013. № 2 (31). С. $156-168$

УДК 539.42

\title{
АСИМПТОТИЧЕСКИЕ МЕТОДЫ НЕЛИНЕЙНОЙ МЕХАНИКИ РАЗРУШЕНИЯ: РЕЗУЛЬТАТЫ, СОВРЕМЕННОЕ СОСТОЯНИЕ И ПЕРСПЕКТИВЫ
}

\author{
Л. В. Степанова, Е. М. Адълина \\ Самарский государственный университет, \\ Россия, 443011, Самара, ул. Академика Павлова, 1. \\ E-mails: lst@ssu.samara.ru, kateadulina@mail.ru
}

\begin{abstract}
Дан краткий обзор основных результатов нелинейной механики разрушения, полученных за последнее десятилетие с помощъю асимптотического анализа и методов возмущений, и обсуждаются асимптотические решения иелого класса задач о трещинах: проблема определения напряжённо-дебормированного состояния в окрестности вершины трещины в условиях смешанного нагружения; задача об усталостном распространении трещины с учётом накопления микроповреждений; проблема определения спектра собственных значений в разложениях по собственным функииям напряжений вблизи вершины трещины и углового выреза, задача построения высших приближений для компонент тензора напряжений и связанная с ней проблема нахождения амплитудных коэбфициентов, зависящих от геометрии рассматриваемого образиа и системы приложенных нагрузок.
\end{abstract}

Ключевые слова: асимптотические методъ, нелинейная механика разрушения, поле напряжений вблизи кончика трещины, нелинейные задачи на собственные значения.

1. Асимптотический анализ в линейной механике разрушения. Асимптотический анализ распределений напряжений, деформаций и перемещений вблизи вершины трещины является одной из фундаментальных задач механики трещин [1]. Вершина трещины может находиться в упругом материале, пластической зоне или зоне, занятой деформациями ползучести; кончик трещины может принадлежать линии раздела нескольких изотропных или анизотропных материалов [2]. Все перечисленные факторы усложняют определение механических полей вблизи устья трещины, и до настоящего времени многие вопросы, связанные с нахождением напряжённо-деформированного состояния в нелинейной механике разрушения, остаются открытыми. Так, в настоящее время в механике трещин и в целом в механике разрушения сложилось понимание процесса разрушения как процесса многомасштабного и многоуровневого, для описания основных закономерностей которого следует прибегать к многомасштабным моделям [3-5]. В рамках многоуровневого подхода процесс разрушения моделируется различными соотношениями на различных расстояниях от вершины трещины с помощью введения многих масштабов. При построении решения задачи в целом представления, работающие на разных расстояниях от кончика трещины, сращиваются в зонах, где справедливы асимптотики соседних областей. Для понимания многоуровневого или многомасштабного подхода целесообразно обратиться к исходным математическим моделям, используемым как в теории механики трещин, так

Лариса Валентиновна Степанова (д.ф.-м.н., доц.), профессор, каф. математического моделирования в механике. Екатерина Михайловна Адылина, аспирант, каф. математического моделирования в механике. 
и в инженерной практике. Одним из наиболее распространенных математических методов построения распределений напряжений, деформаций и перемещений у кончика трещин является асимптотический анализ и подходы, развитые в асимптотической теории $[6,7]$. В рамках линейной теории упругости с помощью методов теории возмущений построено большое количество решений, ставших классическими в механике хрупкого разрушения. Поэтому, обращаясь к современным методам оценки напряжённо-деформированного состояния у вершины трещины в материалах с нелинейными определяющими уравнениями, естественно сначала обратиться к результатам асимптотического анализа линейной теории упругости, ибо результаты, полученные посредством асимптотических методов в линейной теории упругости, служат теоретическим основанием инженерной практики.

В различных точных решениях линейной теории упругости обнаруживается сингулярный характер распределения напряжений и перемещений. Сингулярный характер распределения напряжений появляется при решении краевых задач линейной теории упругости для таких областей, как двугранный угол, для областей, содержащих линию раздела двух и более сред. С физической точки зрения сингулярность поля напряжений соответствует тем зонам, в которых наблюдается концентрация напряжений, приводящая к необратимым деформациям (к развитию пластического течения и деформаций ползучести) и, в конечном итоге, к разрушению элемента конструкции или образца. Вследствие указанных причин окрестность вершины трещины или углового выреза стала предметом интенсивных исследований с конца 18 века.

По всей видимости, первый пример сингулярности поля напряжений в линейной теории упругости дает задача о сосредоточенной силе, действующей на прямолинейную границу бесконечно большой линейной упругой пластины (задача Фламана) [8,9]. Существует фундаментальное решение задачи Фламана, называемое простым радиальным распределением напряжений. Любой элемент $C$, расположенный на расстоянии $r$ от точки приложения сосредоточенной силы, подвергается простому сжатию в радиальном направлении. Компоненты тензора напряжений и поле перемещений определяются при этом выражениями

$$
\sigma_{r r}=-2 P \cos \theta /(\pi r), \quad \sigma_{\theta \theta}=\sigma_{r \theta}=0, \quad u_{r}(\theta=0)=(2 P /(\pi E)) \ln r+B .
$$

Важной особенностью решения задачи Фламана является сингулярность как поля напряжений, так и поля деформаций в точке приложения нагрузки. Более того, эта особенность поля напряжений и перемещений ведет к неинтегрируемой особенности энергии деформации:

$$
W=\lim _{\varepsilon \rightarrow 0}\left(\int_{-\pi / 2}^{\pi / 2} \int_{\varepsilon}^{R} \frac{\sigma_{r r}^{2}}{2 E} r d r\right)=\lim _{\varepsilon \rightarrow 0}\left(\frac{P^{2}}{\pi E} \ln \frac{R}{\varepsilon}\right) .
$$

Поле напряжений (1) обладает особенностью вида $1 / r$ при $r \rightarrow 0$. Математически решение (1) краевой задачи линейной теории упругости о сосредоточенной силе, действующей на прямолинейной границе, приводит к парадоксу, поскольку это решение удовлетворяет всем фундаментальным уравнениям линейной теории упругости, однако в то же время нарушает упрощающие предположения, принимаемые в линейной теории упругости: напряжения не 
должны превышать предела пропорциональности материала; градиенты перемещений должны быть малы; нагрузки должны действовать на недеформируемой поверхности в течение всего процесса нагружения. Для преодоления указанных сложностей предполагается, что малая область материала, примыкающая к точке приложения нагрузки, деформируется пластически и наблюдается пластическое течение вблизи точки приложения нагрузки.

Другая особенность задачи Фламана заключается в том, что ее решение послужило основой для дальнейшего развития асимптотического анализа полей напряжений в линейно упругих телах. В 1907 г. К. Вигхардт впервые обратился к задаче определения поля напряжений вблизи углового выреза, нагруженного двумя сосредоточенными силами. Именно К. Вигхардт, решая обобщенную задачу Фламана, обнаружил факторизацию поля напряжений: поле напряжений может быть представлено в виде функции, представляющей собой произведение двух функций, одна из которых зависит от $r$, тогда как другая является функцией полярного угла $\theta$. Для случая трещины К. Вигхардт обнаружил корневую особенность поля напряжений и начал дискуссию, касающуюся обоснования сингулярности поля напряжений у вершины трещины в линейно упругом материале.

В 1933 г. Дж. Братц опубликовал статью [10], в которой найдено поле напряжений у вершины острого выреза. В то время, когда работа K. Вигхарда была полностью забыта, Дж. Братц воспользовался идеей фон Кармана о введении функции напряжения Эри. Дж. Братц продемонстрировал возможность определения поля напряжений в упругом полупространстве (в двумерной постановке задачи) с общим распределением нормальных и касательных усилий, заданных на границе упругой области. Дж. Братц обобщил подход фон Кармана, показав, что метод может быть применим к любой клиновидной области, нагруженной нормальными и касательными усилиями на прямолинейных границах. В 1933 г. Вестергаард опубликовал работу, посвященную анализу напряжений у вершины трещины в бетонных элементах конструкций в случае изгиба. В его статье, являющейся, по всей видимости, одной из первых попыток использования механики разрушения для описания процесса разрушения квазихрупких материалов, было предложено следующее выражение для функции напряжений Эри:

$$
\Phi=\sum_{m=1}^{\infty} \frac{K_{m}}{2\left(m+\frac{1}{2}\right)\left(m+\frac{3}{2}\right)}\left[\left(m-\frac{1}{2}\right) \sin \left(m+\frac{1}{2}\right) \theta-\left(m+\frac{3}{2}\right) \sin \left(m-\frac{1}{2}\right) \theta\right] .
$$

Несмотря на упомянутые пионерские работы в области применения асимптотических методов в линейной механике разрушения, в полной мере возможность разделения переменных в решении задач для клиновидных областей была раскрыта М. Уильямсом, предложившим метод разложения по собственным функциям функции напряжений Эри, компонент тензора напряжений и вектора перемещений. Именно в его работах $[11,12]$ функция напряжений Эри была представлена в форме произведения двух функций, одна из которых зависела от радиальной переменной $r(r$-расстояние от вершины клина до рассматриваемой точки), а вторая - от полярного угла $\theta$ :

$$
\Phi(r, \theta)=\sum r^{\lambda_{j}+1} f_{j}\left(\theta, \lambda_{j}\right)
$$

где $\lambda_{j}$ и $f_{j}(\theta)$ - собственные значения и соответствующие им собственные 
функции, определяемые из решения задач на собственные значения. Сравнительная простота и легкость использования представления (2) при решении краевых задач механики трещин были важным аспектом, который обусловил широкое распространение этого подхода [7]. Следует отметить, что, с другой стороны, природа разделения переменных в решении задач, сводящихся к бигармоническому уравнению, была уже установлена в механике линейновязкой жидкости при изучении двумерного течения Стокса - течения вязкой несжимаемой жидкости, которое может быть описано посредством функции тока $\Psi$, удовлетворяющей бигармоническому уравнению. В механике жидкости и газа применительно к бигармоническому уравнению относительно функции тока метод разложения по собственным функциям был предложен Дином и Монтагоном [13]. Они приложили метод разложения по собственным функциям к задачам о движении линейно-вязкой жидкости вблизи угловых областей, что с математической точки зрения аналогично решению задач определения напряжённо-деформированного состояния вблизи угловых вырезов в линейно-упругих материалах. Упомянутые работы, посвященные построению асимптотик механических полей близи трещин и угловых вырезов, обеспечили основу для ответа на фундаментальные вопросы механики трещин: при каких условиях трещина получает возможность распространяться и продолжает расти? К. Вигхардт в своей работе сформулировал следующие вопросы: при каком значении сосредоточенной силы $P$ для материала с заданными упругими и прочностными свойствами начнется разрушение материала, в каком месте и в каком направлении будет развиваться разрушение?

В современной нелинейной механике разрушения одним из наиболее распространенных методов анализа напряжённо-деформированного состояния вблизи вершины трещины как в линейно-упругих материалах, так и в материалах с нелинейными определяющими уравнениями, является метод разложения по собственным функциям, восходящий к работам М. Уильямса $[11,12]$, в которых впервые было использовано разложение функции напряжений Эри в ряд по степеням расстояния от кончика трещины или вершины углового выреза в линейно-упругом материале (разложение по собственным функциям). В ставших классическими работах Дж. Райса, Дж. Розенгрена и Дж. Хатчинсона [14-16] асимптотическое представление решения задачи определения напряжённо-деформированного состояния в окрестности вершины трещины в материале с определяющими уравнениями Рамберга-Осгуда $\varepsilon=\sigma / E+\alpha \sigma^{n}$ разыскивалось в форме

$$
\begin{array}{ll}
\sigma_{i j}(r, \theta)=K r^{-1 /(n+1)} \tilde{\sigma}_{i j}(\theta), & \sigma_{e}(r, \theta)=K r^{-1 /(n+1)} \tilde{\sigma}_{e}(\theta), \\
\varepsilon_{i j}^{p}(r, \theta)=\alpha K^{n} r^{-n /(n+1)} \tilde{\varepsilon}_{i j}(\theta), & u_{i}(r, \theta)=\alpha K^{n} r^{1 /(n+1)} \tilde{u}_{i}(\theta) .
\end{array}
$$

С тех пор асимптотический анализ распределения напряжений у кончика трещины стал неотъемлемой частью исследований, проводимых в механике разрушения $[2,17]$. Только в самое последнее время метод разложения по собственным функциям полей напряжений и перемещений у вершины трещины или углового выреза был применен в целом ряде исследований $[18,19]$.

Асимптотическое представление решения в форме (3) широко используется в нелинейной механике разрушения для анализа полей напряжений, деформаций и перемещений в условиях смешанного нагружения [20-22]. Первое обращение к исследованию смешанных форм деформирования, по всей види- 
мости, было сделано в работах Си $[23,24]$, где рассмотрены поля напряжений и деформаций вблизи вершины трещины, находящейся под действием растягивающей и сдвиговой нагрузок (т.о. рассматривалось смешанное нагружение, отвечающее трещинам типа I и типа II). Си впервые ввёл коэффициент смешанности нагружения:

$$
M^{p}=\frac{2}{\pi} \operatorname{arctg}\left|\lim _{r \rightarrow 0} \frac{\sigma_{\theta \theta}(r, \theta=0)}{\sigma_{r \theta}(r, \theta=0)}\right| .
$$

Параметр смешанности нагружения $M^{p}$ принимает значение $M^{p}=0$ для чистого поперечного сдвига и значение $M^{p}=1$ для нормального отрыва; $0<M^{p}<1$ для всех промежуточных типов приложенной нагрузки.

В [23] приведены распределения напряжений, деформаций и перемещений у вершины трещины в материале, который подчиняется степенному закону деформационной теории пластичности для отдельных значений параметра смешанности нагружения в условиях плоской деформации. Там же могут быть найдены контуры областей пластического течения у вершины трещины для различных значений показателя нелинейности материала и параметра смешанности нагружения. Подробный анализ напряжённо-деформированного состояния у вершины наклонной трещины для степенного определяющего закона выполнен в [25] (подход, используемый авторами, также изложен в [26]). В этой работе авторами предлагается процедура решения краевой задачи для нелинейного дифференциального уравнения, получающейся из проблемы нахождения полей напряжений и деформаций у вершины трещины в условиях смешанного нагружения. В [27] собраны существенные результаты, полученные для смешанных форм деформирования к 2003 году.

Расчёты угловых распределений напряжений и деформаций у вершины трещины в материале со степенным определяющим законом для различных значений параметра смешанности нагружения для плоского деформированного состояния приведены в [22] в полном диапазоне смешанных форм деформирования - от нормального отрыва до чистого сдвига.

2. Смешанное нагружение элемента конструкции с трещиной (нормальный отрыв и поперечный сдвиг) в условиях плоского напряжённого состояния. Рассмотрим стационарную трещину в материале со степенным законом упрочнения. Введём прямоугольную декартову систему координат с центром в вершине трещины и полярную систему координат $(r, \theta)$ с полюсом в вершине трещины. Уравнения равновесия представляются в форме

$$
r \sigma_{r r, r}+\sigma_{r \theta, \theta}+\left(\sigma_{r r}-\sigma_{\theta \theta}\right)=0, \quad \sigma_{\theta \theta, \theta}+r \sigma_{r \theta, r}+2 \sigma_{r \theta}=0 .
$$

Условие совместности деформаций формулируется как

$$
2\left(r \varepsilon_{r \theta}, \theta\right),_{r}=\varepsilon_{r r}, \theta \theta-r \varepsilon_{r r}, r+r\left(r \varepsilon_{\theta \theta}\right),_{r r} .
$$

Определяющие уравнения $\varepsilon_{i j}=3 B \sigma_{e}^{n-1} s_{i j} / 2$ для случая плоского напряжённого состояния принимают форму

$$
\begin{gathered}
\varepsilon_{r r}=B \sigma_{e}^{n-1}\left(2 \sigma_{r r}-\sigma_{\theta \theta}\right) / 2, \quad \varepsilon_{\theta \theta}=B \sigma_{e}^{n-1}\left(2 \sigma_{\theta \theta}-\sigma_{r r}\right) / 2, \\
\varepsilon_{r \theta}=3 B \sigma_{e}^{n-1} \sigma_{r \theta} / 2, \quad \sigma_{e}^{2}=\sigma_{r r}^{2}+\sigma_{\theta \theta}^{2}-\sigma_{r r} \sigma_{\theta \theta}+3 \sigma_{r \theta}^{2} .
\end{gathered}
$$


Граничные условия задачи есть условия отсутствия поверхностных усилий на берегах трещины $\sigma_{\theta \theta}(r, \theta= \pm \pi)=0, \sigma_{r \theta}(r, \theta= \pm \pi)=0$.

3. Асимптотическое представление компонент тензора напряжений и деформаций вблизи вершины трещины. Будем искать асимптотическое представление функции напряжений Эри в окрестности вершины трещины в форме $F(r, \theta)=r^{\lambda+2} f(\theta)$. Тогда компоненты тензора напряжений принимают вид

$$
\begin{gathered}
\sigma_{\theta \theta}(r, \theta)=r^{\lambda}(\lambda+2)(\lambda+1) f(\theta), \quad \sigma_{r r}(r, \theta)=r^{\lambda}\left[(\lambda+2) f(\theta)+f^{\prime \prime}(\theta)\right], \\
\sigma_{r \theta}(r, \theta)=-r^{\lambda}(\lambda+1) f^{\prime}(\theta) .
\end{gathered}
$$

Асимптотическое представление интенсивности напряжений в окрестности вершины трещины может быть представлено как

$$
\begin{aligned}
\sigma_{e}(r, \theta)=r^{\lambda} f_{e}(\theta), \quad f_{e}^{2}=[ & \left.(\lambda+2) f+f^{\prime \prime}\right]^{2}+[(\lambda+2)(\lambda+1) f]^{2}- \\
& -\left[(\lambda+2) f+f^{\prime \prime}\right](\lambda+2)(\lambda+1) f+3(\lambda+1)^{2} f^{2} .
\end{aligned}
$$

В силу соотношений (5) компоненты тензора деформаций в окрестности вершины трещины определяются выражениями

$$
\varepsilon_{r r}=B r^{\lambda n} \tilde{\varepsilon}_{r r}(\theta), \quad \varepsilon_{\theta \theta}=B r^{\lambda n} \tilde{\varepsilon}_{\theta \theta}(\theta), \quad \varepsilon_{r \theta}=B r^{\lambda n} \tilde{\varepsilon}_{r \theta}(\theta),
$$

где приняты следующие обозначения:

$$
\begin{gathered}
\tilde{\varepsilon}_{r r}=f_{e}^{n-1}\left[(\lambda+2)(1-\lambda) f+2 f^{\prime \prime}\right] / 2, \quad \tilde{\varepsilon}_{\theta \theta}=f_{e}^{n-1}\left[(\lambda+2)(2 \lambda+1) f-f^{\prime \prime}\right] / 2, \\
\tilde{\varepsilon}_{r \theta}(\theta)=-3 f_{e}^{n-1}(\lambda+1) f^{\prime}(\theta) / 2 .
\end{gathered}
$$

Выражения (6) для компонент тензора деформаций позволяют получить нелинейное обыкновенное дифференциальное уравнение для функции $f(\theta)$ :

$$
2(\lambda n+1) \tilde{\varepsilon}_{r \theta, \theta}=\tilde{\varepsilon}_{r r}, \theta \theta-\lambda n \tilde{\varepsilon}_{r r}+(\lambda n+1) \lambda n \tilde{\varepsilon}_{\theta \theta}
$$

или

$$
\begin{gathered}
f^{I V} f_{e}^{2}\left\{(n-1)\left[2 f^{\prime \prime}+s(3-s) f\right]^{2} / 2+2 f_{e}^{2}\right\}+6(\lambda+1)(\lambda n+1)(n-1) f_{e}^{2} f^{\prime} \times \\
\times\left\{\left(s f+f^{\prime \prime}\right)\left(s f^{\prime}+f^{\prime \prime \prime}\right)+s^{2}(s-1)^{2} f f^{\prime}-s(s-1)\left(s f^{\prime}+f^{\prime \prime \prime}\right) f / 2-\right. \\
\left.-s(s-1)\left(s f+f^{\prime \prime}\right) f^{\prime} / 2+3(s-1)^{2} f^{\prime} f^{\prime \prime}\right\}+ \\
+6(\lambda+1)(\lambda n+1)(n-1) f_{e}^{4} f^{\prime \prime}+(n-1)(n-3)\left[2\left(s f+f^{\prime \prime}\right)-s(s-1) f\right] \times \\
\times\left\{\left(s f+f^{\prime \prime}\right)\left(s f^{\prime}+f^{\prime \prime \prime}\right)+s^{2}(s-1)^{2} f f^{\prime}-s(s-1)\left(s f^{\prime}+f^{\prime \prime \prime}\right) f / 2-\right. \\
\left.-s(s-1)\left(s f+f^{\prime \prime}\right) f^{\prime} / 2+3(s-1)^{2} f^{\prime} f^{\prime \prime}\right\}^{2}+ \\
+(n-1) f_{e}^{2}\left[2 f^{\prime \prime}+s(3-s) f\right]\left\{\left(s f^{\prime}+f^{\prime \prime \prime}\right)^{2}+\left(s f+f^{\prime \prime}\right) s f^{\prime \prime}+\right. \\
+s^{2}(s-1)^{2}\left(f^{\prime 2}+f f^{\prime \prime}\right)-s^{2}(s-1) f f^{\prime \prime} / 2-s(s-1)\left(s f^{\prime}+f^{\prime \prime \prime}\right) f^{\prime}- \\
\left.-s(s-1)\left(s f+f^{\prime \prime}\right) f^{\prime \prime} / 2+3(s-1)^{2}\left(f^{\prime \prime 2}+f^{\prime} f^{\prime \prime \prime}\right)\right\}+ \\
+2(n-1) f_{e}^{2}\left[2 f^{\prime \prime \prime}+s(3-s) f^{\prime}\right]\left\{\left(s f+f^{\prime \prime}\right)\left(s f^{\prime}+f^{\prime \prime \prime}\right)+s^{2}(s-1)^{2} f f^{\prime}-\right. \\
\left.-s(s-1)\left(s f^{\prime}+f^{\prime \prime \prime}\right) f / 2-s(s-1)\left(s f+f^{\prime \prime}\right) f^{\prime} / 2+3(s-1)^{2} f^{\prime} f^{\prime \prime}\right\}+ \\
+f_{e}^{4}(3-s) s f^{\prime \prime}-\lambda n f_{e}^{4}\left[2 f^{\prime \prime}+s(3-s) f\right]+ \\
\quad+(\lambda n+1) \lambda n f_{e}^{4}\left[s(2 s-3) f-f^{\prime \prime}\right]=0 .
\end{gathered}
$$


Решение уравнения (7) должно удовлетворять условиям, следующим из требований отсутствия поверхностных усилий на берегах трещины: $f(\theta=$ $= \pm \pi)=0, f^{\prime}(\theta= \pm \pi)=0$.

Вместе с этим необходимо учесть условия, задающие вид смешанного нагружения, которые формулируются на линии продолжения трещины $(\theta=0)$. Из определения параметра смешанности нагружения (4) следует

$$
f^{\prime}(\theta=0)=-(\lambda+2) f(\theta=0) / \operatorname{tg}\left(\pi M^{p} / 2\right),
$$

что позволяет сформулировать начальную задачу для отрезка $[0, \pi]$. Необходимо найти решение уравнения (7), удовлетворяющее условиям

$$
f(0)=1, \quad f^{\prime}(0)=-(\lambda+2) / \operatorname{tg}\left(\pi M^{p} / 2\right), \quad f^{\prime \prime}(0)=A_{2}, \quad f^{\prime \prime \prime}(0)=A_{3},
$$

где неизвестные на данном этапе построения решения константы $A_{2}$ и $A_{3}$ подбираются таким образом, чтобы выполнялись краевые условия на берегу трещины: $f(\pi)=0, f^{\prime}(\pi)=0$.

Константы $A_{2}$ и $A_{3}$ были найдены для всех значений показателя упрочнения материала $n$ от 2 до 13 и для всех значений параметра смешанности нагружения $0 \leqslant M^{p} \leqslant 1$. Затем осуществлялось построение решения уравнения (7) на отрезке $[-\pi, 0]$. С целью построения решения на этом отрезке формулировалась начальная задача с условиями при $\theta=-\pi$ :

$$
f(-\pi)=0, \quad f^{\prime}(-\pi)=0, \quad f^{\prime \prime}(-\pi)=B_{2}, \quad f^{\prime \prime \prime}(-\pi)=B_{3} .
$$

Постоянные $B_{2}$ и $B_{3}$ находятся из условий равновесия элемента, расположенного на луче $\theta=0$, которые в терминах функции $f(\theta)$ имеют вид

$$
f^{-}(\theta=0)=f^{+}(\theta=0), \quad\left(f^{\prime}\right)^{-}(\theta=0)=\left(f^{\prime}\right)^{+}(\theta=0) .
$$

Константы $B_{2}$ и $B_{3}$ были найдены для всех значений показателя упрочнения материала $n$ от 2 до 13 и для всех значений параметра смешанности нагружения $0 \leqslant M^{p} \leqslant 1$.

Угловые распределения компонент тензоров напряжений и деформаций в окрестности вершины трещины в условиях плоского напряжённого состояния для различных значений параметра смешанности нагружения для $n=5$ показаны на рис. 1, 2.

Можно выделить основные вопросы, остающиеся открытыми в нелинейной механике разрушения и требующие пристального внимания в силу их существенной значимости как для развития теории, так и с точки зрения инженерных приложений.

4. Асимптотический анализ в нелинейной механике разрушения. Построение высших приближений в асимптотических разложениях компонент тензора напряжений, деформаций и вектора перемещений. В последнее время в нелинейной механике разрушения, одной из задач которой является определение напряжённо-деформированного состояния в непосредственной окрестности вершины трещины, большое внимание уделяется построению высших приближений в асимптотических разложениях механических величин (полей напряжений, деформаций и перемещений) в окрестности вершины трещины 

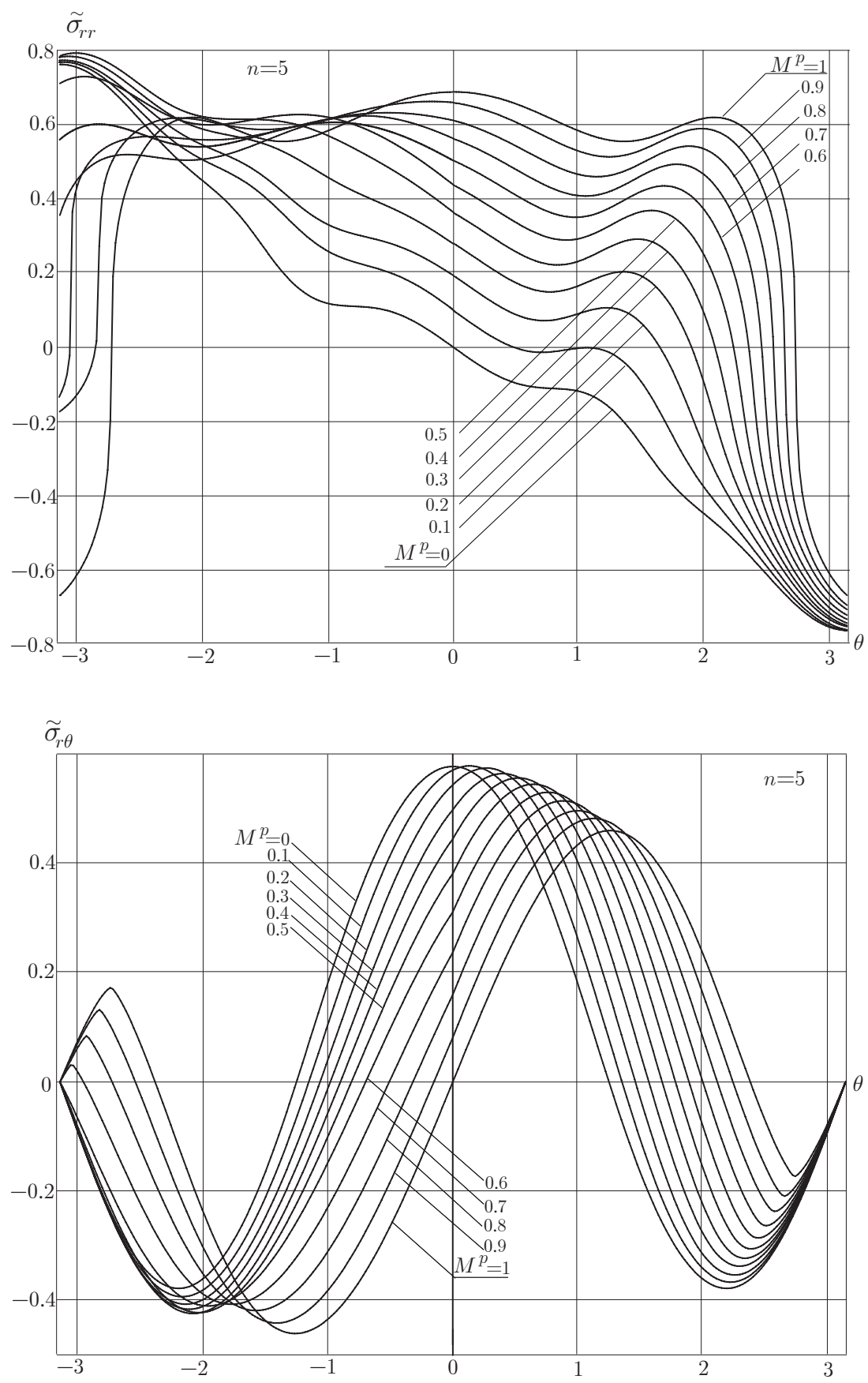

Рис. 1. Угловые распределения компонент тензора напряжений $\sigma_{r r}, \sigma_{r \theta}$ для $n=5$ 

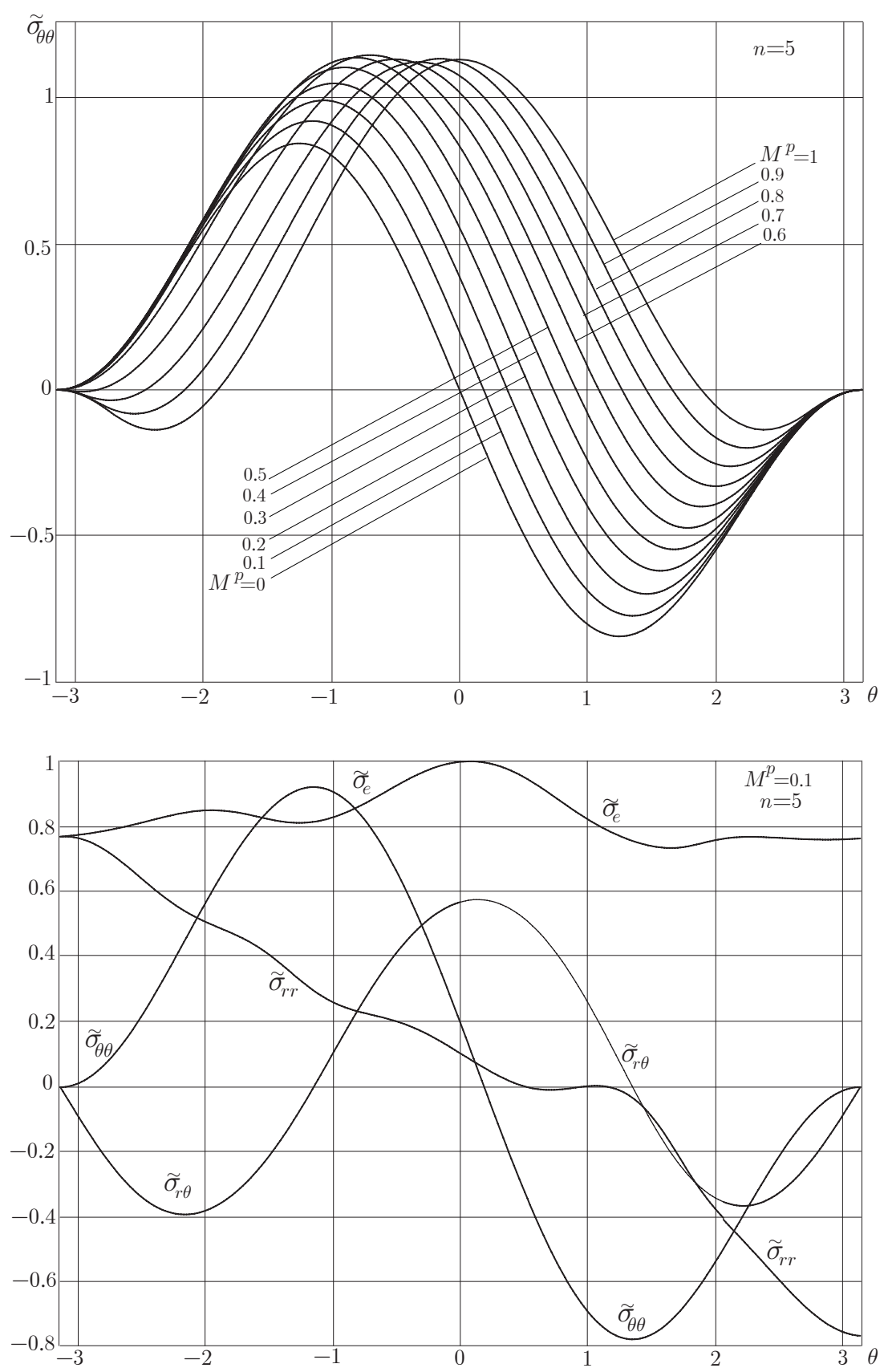

Рис. 2. Угловые распределения компонент тензора напряжений $\sigma_{\theta \theta}$ для $n=5$ 
в материалах с нелинейными определяющими уравнениями. Для материалов, следующих степенному закону теории установившейся ползучести $\dot{\varepsilon}_{i j}=$ $=3 B \sigma_{e}^{n-1} s_{i j} / 2$, асимптотические разложения компонент тензора напряжений в непосредственной окрестности вершины трещины $(r \rightarrow 0)$ могут быть представлены в форме

$$
\sigma_{i j}(r, \theta)=\left(\frac{C^{*}}{B I_{n} r}\right)^{1 /(n+1)} \sigma_{i j}^{(1)}(\theta)+A_{2} r^{s_{2}} \sigma_{i j}^{(2)}(\theta)+A_{3} r^{s_{3}} \sigma_{i j}^{(3)}(\theta)+\ldots,
$$

где $C^{*}$ - интеграл, определяемый равенством

$$
C^{*}=\int_{\Gamma}\left(\dot{W} d x_{2}-\sigma_{i j} n_{j} \frac{\partial \dot{u}_{i}}{\partial x_{1}} d s\right), \quad \dot{W}=\int_{0}^{\dot{\varepsilon}_{k l}} \sigma_{i j} d \dot{\varepsilon}_{i j}=\frac{n}{n+1} \sigma_{e} \dot{\varepsilon}_{e} .
$$

В литературе имеется достаточно большое число оценок инвариантного $C^{*}$-интеграла, полученных для различных образцов, наиболее часто используемых в эксперименте. Однако остаются открытыми многие вопросы, связанные с определением второго коэффициента в асимптотических разложениях (8), а именно $A_{2}$. Естественно предположить, что $A_{2}$ в общем случае является функцией приложенных нагрузок, геометрических характеристик образца и показателя нелинейности материала $n$. Определение этого коэффициента и составляет задачу теории, поскольку даже известные оценки, полученные для первого амплитудного коэффициента и приведенные в справочной литературе [28], дают существенную погрешность. Например, для образца с односторонним боковым надрезом в [28] для инвариантного $J$-интеграла приведена формула

$$
J=B \sigma_{0} \varepsilon_{0} W(1-a) a h_{1}\left(P / P_{0}\right),
$$

где $h_{1}$ - безразмерная функция приложенной нагрузки и геометрии образца. Оказывается, что уточненные вычисления, проведенные в последнее время, показали, что значения функции $h_{1}$ могут отличаться от приведенных в [28] более чем на сто процентов. Следовательно, с появлением вычислительных комплексов, таких как SIMULIA Abaqus, предоставляется возможность получения более точных оценок для параметров механики разрушения.

5. Нелинейные задачи на собственные значения. Метод разложения по собственным функциям механических величин в механике разрушения приводит к нелинейным задачам на собственные значения, которые решаются, как правило, численно. Метод возмущений позволяет найти аналитическое решение нелинейных задач на собственные значения, возникающих в нелинейной механике разрушения. Метод возмущений для построения аналитического решения задачи определения напряжённо-деформированного состояния и поля сплошности у растущей в условиях циклического нагружения трещины был использован в [29], где показывается, что метод разложения по собственным функциям, когда функция напряжений Эри и параметр сплошности разыскиваются в форме $F(r, \theta)=r^{\lambda} f(\theta), \psi(r, \theta)=r^{\mu} g(\theta)$, редуцирует задачу к нелинейной задаче на собственные значения. Обычно в механике разрушения для построения решения нелинейных задач на собственные значения прибегают к численному интегрированию уравнений задачи, что может привести к 
ошибочным результатам. Для преодоления указанных сложностей при решении нелинейной задачи на собственные значения в ходе применения метода возмущений построены четырехчленные асимптотические разложения искомых функций. В [29] получены аккуратные выражения для искомых функций вплоть до слагаемых порядка $\mathrm{O}\left(\varepsilon^{4}\right)$, где $\varepsilon=\mu-\mu_{0}-$ разность между собственными значениями, отвечающими нелинейной и линейной задачам:

$$
\begin{aligned}
& n=1+\varepsilon n_{1}+\varepsilon^{2} n_{2}+\varepsilon^{3} n_{3}+\varepsilon^{4} n_{4}+\ldots, \\
& m=1+\varepsilon m_{1}+\varepsilon^{2} m_{2}+\varepsilon^{3} m_{3}+\varepsilon^{4} m_{4}+\ldots, \\
& f(\theta)=f_{0}(\theta)+\varepsilon f_{1}(\theta)+\varepsilon^{2} f_{2}(\theta)+\varepsilon^{3} f_{3}(\theta)+\ldots, \\
& g(\theta)=g_{0}(\theta)+\varepsilon g_{1}(\theta)+\varepsilon^{2} g_{2}(\theta)+\varepsilon^{3} g_{3}(\theta)+\ldots .
\end{aligned}
$$

Рассматриваемый подход, базирующийся на введении малого параметра, позволяет найти весь спектр собственных значений:

$$
\lambda=\mu=\frac{1}{1+n-m} .
$$

\section{6. Направления дальнейшего развития асимптотических методов в нели-} нейной механике разрушения Одними из важных проблем являются разработка и развитие математических многомасштабных моделей накопления повреждений на макро-, мезо- и микроуровнях у вершины трещины в элементах конструкций, находящихся в условиях пластического течения, высокотемпературной ползучести, усталостного нагружения, а также формулировка критериев разрушения неупругих материалов, учитывающих процессы повреждаемости материала в процессе деформирования [3-5]. Многомасштабность или многоуровневость процесса разрушения можно отразить, вводя в рассмотрение поля напряжений на различных расстояниях от кончика трещины с разной особенностью (с различной степенью сингулярности). Таким образом, сильная и слабая особенности механических полей у вершины трещины отражают многомасштабный процесс разрушения. В силу этих особенностей моделирования процесса разрушения представляется важным знание всех возможных решений задачи определения напряжённо-деформированного состояния у вершины трещины, а не только тех слагаемых в асимптотических разложениях полей напряжений и перемещений, которые традиционно удерживаются в классических решениях. Таким образом, многоуровневые модели разрушения требуют исследования всего спектра собственных значений в задаче определения полей напряжений и перемещений в окрестности вершины трещины в материалах с нелинейными определяющими уравнениями. Если для упругих материалов в линейной механике разрушения построено полное разложение (решение Уильямса $[11,12]$ ), то для материалов со степенными определяющими соотношениями многие вопросы остаются открытыми.

Работа выполнена при поддержке РФФИ (проект № 12-08-00390-a).

\section{БИБЛИОГРАФИЧЕСКИЙ СПИСОК}

1. H. D. Bui, Fracture Mechanics: Inverse Problems and Solutions / Solid Mechanics and Its Applications. Vol. 139. Dordrecht: Springer, 2006. xxiii+398 pр.; русск. пер.: Х. Д. Бъюи, Механика разрушения: Обратные задачи и решения / пер. с англ. Л. В. Степановой. Москва: Физматлит, 2011. 412 с.

2. J. Li, N. Recho, Méthodes asymptotiques en mécanique de la rupture. Paris: HermèsLavoisier, 2002. 262 pp. 
3. G. C. Sih, "Crack tip mechanics based on progressive damage of arrow: Hierarchy of singularities and multiscale segment" // J. Theoret. Appl. Fract. Mech., 2009. Vol. 51, no. 1. Pp. 11-32.

4. G. C. Sih, X. S. Tang, "Simultaneity of multiscaling for macro-meso-micro damage model represented by strong singularities" // J. Theoret. Appl. Fract. Mech., 2004. Vol.42, no. 3. Pp. 199-225.

5. G. C. Sih, X. S. Tang, "Weak and strong singularities reflecting multiscale damage: microboundary conditions for free-free, fixed-fixed and free-fixed constraints" // J. Theoret. Appl. Fract. Mech., 2005. T. 43, № 1. C. 5-62.

6. И. И. Аргатов, Введение в асимптотическое моделирование в механике. СПб.: Политехника, 2004. 302 с. [I. I. Argatov, Introduction to Asymptotic Modelling in Mechanics. St. Petersburg: Polytechnics, 2004. 302 pp.]

7. Л. В. Степанова, Математические методы механики разрушения. М.: Физматлит, 2009. 336 c. [L. V. Stepanova, Mathematical methods of fracture mechanics. Moscow: Fizmatlit, 2009. 336 pp.]

8. A. Flamant, "Sur la répartition des pressions dans un solide rectangulaire chargé transversalement" // Compte. Rendu. Acad. Sci. Paris, 1892. Vol. 114. Pp. 1465-1468.

9. S. P. Timoshenko, J. N. Goodier, Theory of elasticity. New York: McGraw-Hill, 1970. 567 рр.; русск. пер.: С. П. Тимошенко, Дж. Гудъер, Теория упругости. М.: Наука, 1979. $560 \mathrm{c}$.

10. J. H. A. Brahtz, "Stress distribution in a reentrant corner" // Trans. Amer. Soc. Mech. Eng., 1933. Vol. 55. Pp. 31-71.

11. M. L. Williams, "Stress singularities resulting from various boundary conditions in angular corners of plates in extension" // J. Appl. Mech., ASME, 1952. Vol. 74. Pp. 526-528.

12. M. L. Williams, "On the stress distribution at the base of a stationary crack" // J. Appl. Mech., ASME, 1957. Vol. 24. Pp. 109-114.

13. W. R. Dean, P. E. Montagnon, "On the steady motion of viscous liquid in a corner"// Proc. Cambridge Philos. Soc., 1948. no. 45. Pp. 389-395.

14. J. M. Hutchinson, "Singular behaviour at the end of tensile crack in a hardening material" // J. Mech. Phys. Solids, 1968. Vol. 16, no. 1. Pp. 13-31.

15. J. M. Hutchinson, "Plastic stress and strain fields at a crack tip" // J. Mech. Phys. Solids, 1968. Vol. 16, no. 5. Pp. 337-347.

16. J. R. Rice, G. F. Rosengren, "Plane strain deformation near a crack tip in a power-law hardening material" // J. Mech. Phys. Solids, 1968. Vol. 16, no. 1. Pp. 1-12.

17. A. Carpinteri, M. Paggi, "Asymptotic analysis in Linear Elasticity: From the pioneering studies by Wieghardt and Irwin until today"// Engn. Fract. Mech., 2009. Vol. 76, no. 12. Pp. 1771-1784.

18. Z. Niu, C. Cheng, N. Recho, "A new boundary element approach of modelling singular stress fields of plane V-notch problems" // Int. J. Solids Struct., 2009. Vol.46, no.16. Pp. 29993008 .

19. G. Hello, M. B. Taha, J. M. Roelandt, "Analytical determination of coefficients in crack-tip stress expansions for a finite crack in an infinite plane medium" // Int. J. Solids Struct., 2012. Vol. 49, no. 3-4. Pp. 556-566.

20. В. Н. Шлянников, "Смешанные моды развития трещин при сложном напряженном состоянии (обзор)" // Завод. лаб., 1990. Т. 56. С. 77-90. [V. N. Shlyannikov, "Mixed-modes crack growth under complex stress strain state (Review)" // Zavod. Lab., 1990. Vol.56. Pp. 77-90].

21. В. Н. Шлянников, Вычислительная механика деформирования и разрушения. Казань: Казан. гос. энерг. ун-т, 2002. 228 с. [V. N. Shlyannikov, Computational Mechanics of Deformation and Fracture. Kazan: Kazan State Power Engn. Univ., 2002. 228 pp.]

22. В. Н. Шлянников, С. Ю. Кислова, "Параметры смешанных форм деформирования для трещины в виде математического разреза" // Изв. Сарат. ун-та. Нов. сер. Сер. Математика. Механика. Информатика, 2009. Т. 9, №1. С. 77-84. [V. N. Shlyannikov, 
S. Yu. Kislova, "Mode mixity parameters for mathematical crack type" // Izv. Saratov. Univ. Mat. Mekh. Inform., 2009. Vol. 9, no. 1. Pp. 77-84].

23. C. F. Shih, Elastic-plastic analysis of combined mode crack problems: $\mathrm{Ph}$. D. Thesis. Cambridge, M.A.: Harvard University, 1973.

24. C. F. Shih, "Small scale yielding analysis of mixed mode plane-strain crack problems" / In: Fracture Analysis: Proceedings of the National Symposium on Fracture Mechanics. Part 2 (College Park, Md., August 27-29, 1973). Philadelphia, Pa.: American Society for Testing and Materials, 1974. 187-210 pp.

25. В. И. Астафъев, А. Н. Крутов, "Распределение напряжений вблизи вершины наклонной трещины в нелинейной механике разрушения" // Изв. РАН. MTT, 2001. № 5. С. 125133; англ. пер.: V. I. Astaf'ev, A. N. Krutov, "Stress distribution near the tip of an inclined crack in the nonlinear fracture mechanics" // Mech. Solids, 2001. Vol. 36, no. 5. Pp. 101-108.

26. Астафъев В.И., Радаев Ю.Н., Степанова Л.В., Нелинейная механика разрушения. Самара: Самарский университет, 2001. 632 с. [V. I. Astaf'ev, Yu. N. Radayev, L. V. Stepanova, Nonlinear Fracture Mechanics. Samara: Samara State Univ., 2001. 632 pp.]

27. V. N. Shlyannikov, Elastic-Plastic Mixed-Mode Fracture Criteria and Parameters / Lecture Notes in Applied and Computational Mechanics. Vol. 7. Berlin: Springer, 2003. 246 pp.

28. V. Kumar, M. D. German, C. F. Shih, An engineering approach for elastic-plastic fracture analysis: General Electric Company, NP-1931, Research Project 1237-1, Topical Report. Schenectady, New York, 1981. 71 pp.

29. Е. М. Адылина, С. А. Игонин, Л. В. Степанова, "О нелинейной задаче на собственные значения, следующей из анализа напряжений у вершины усталостной трещины” // Вестн. СамГУ, 2012. №3/1(94). C. 83-102. [E. M. Adylina, S. A. Igonin, L. V. Stepanova, "About a non-linear task on eigenvalues incurring from the analysis of tensions at the fatigue crack tip" // Vestnik SamGU, 2012. no. 3/1(94). Pp. 83-102].

Поступила в редакцию 10/XI/2012;

в окончательном варианте - 15/XII/2012.

MSC: 74E35, 74K20

\section{ASYMPTOTIC METHODS OF NONLINEAR FRACTURE MECHANICS: RESULTS, CONTEMPORARY STATE AND PERSPECTIVES}

\section{V. Stepanova, E. M. Adylina}

Samara State University,

1, Academician Pavlov st., Samara, 443011, Russia.

E-mails: lst@ssu.samara.ru, kateadulina@mail.ru

In the paper the brief review of the important results of nonlinear fracture mechanics recently obtained by the asymptotic methods and perturbation techniques is given. The asymptotic solutions of the whole class of crack problems are discussed. Applications of the asymptotic analysis for crack problems of nonlinear fracture mechanics such that crack tip fields in damaged medium, fatigue crack growth problems in damaged medium, nonlinear eigenvalue problems arising from fracture mechanics analysis, nonlinear eigenvalue analysis including higher order terms are elucidated.

Key words: asymptotic methods, nonlinear fracture mechanics, crack tip stress field, nonlinear eigenvalue problems.

Original article submitted 10/XI/2012; revision submitted 15/XII/2012.

Larisa V. Stepanova (Dr. Sci. (Phys. \& Math.)), Professor, Dept. of Mathematical Modelling in Mechanics. Ekaterina M. Adylina, Postgraduate Student, Dept. of Mathematical Modelling in Mechanics. 